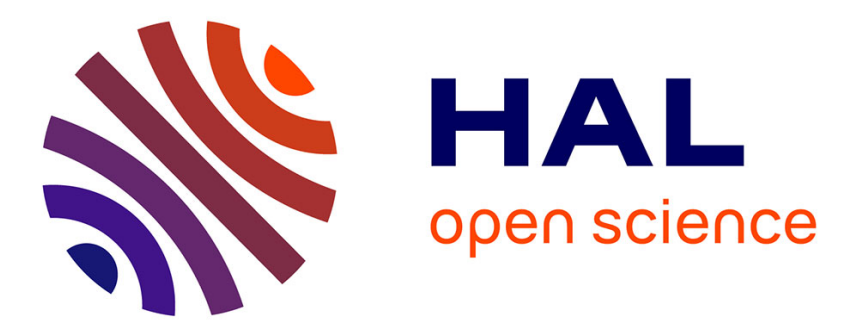

\title{
Le dédoublement résidentiel, descripteur des bifurcations des trajectoires des stations de montagne
} Philippe Bachimon, Pierre Dérioz, Vincent Vlès

\section{To cite this version:}

Philippe Bachimon, Pierre Dérioz, Vincent Vlès. Le dédoublement résidentiel, descripteur des bifurcations des trajectoires des stations de montagne. Vincent Vlès; Christophe Bouneau. Stations en tension, Peter Lang, 2016, 978-2-87574-318-3. hal-01737140

\author{
HAL Id: hal-01737140 \\ https://hal.science/hal-01737140
}

Submitted on 19 Mar 2018

HAL is a multi-disciplinary open access archive for the deposit and dissemination of scientific research documents, whether they are published or not. The documents may come from teaching and research institutions in France or abroad, or from public or private research centers.
L'archive ouverte pluridisciplinaire HAL, est destinée au dépôt et à la diffusion de documents scientifiques de niveau recherche, publiés ou non, émanant des établissements d'enseignement et de recherche français ou étrangers, des laboratoires publics ou privés. 
BACHIMON Ph., DERIOZ Ph., VLES V., 2016. "Le dédoublement résidentiel, descripteur des bifurcations des trajectoires des stations de montagne "1, in VLES V. \& BOUNEAU Ch. (dir), 2016. Stations en tension, Bruxelles : Peter Lang, $260 \mathrm{p}$.

\section{Le dédoublement résidentiel, descripteur des bifurcations des trajectoires des stations de montagne ${ }^{1}$.}

Envisagés comme des systèmes territoriaux localisés (Perret, 1993 ; Moine, 2007 ; Dérioz 2012 : 203-204), structurés autour d'une fonctionnalité touristique ou récréative dominante (Vlès, 1996, 2000, 2006 ; Dérioz et al., 2015), les stations de montagne présentent pour la période contemporaine des trajectoires marquées par un élargissement de l'assise territoriale (François, 2007) qui s'articule avec une diversification fonctionnelle : décrites il y a peu encore comme des exemples achevés de systèmes sociospatiaux spécialisés (Clary, 1983 : 66-86 ; Stock, $2003: 57-70)$, précisément localisés et structurés autour de l'exploitation - économique (organisation des pratiques) et symbolique (communication) - de la " ressource neige ", les stations de sports d'hiver tendent aujourd'hui à se fondre dans des ensembles territoriaux plus étendus, moins nettement circonscrits aussi sans doute, pour lesquels Vincent Vlès (2014 : 23-27) propose de parler de "métastations ». Souvent moins exclusive, l'activité touristique y prend aussi des formes plus variées et moins exclusivement hivernales. Si elles ne sont pas les seules à connaître cette évolution (Boudières et al., 2004 ; Bourdeau, 2009), les stations pyrénéennes n'échappent nullement à cette tendance. Cette mutation progressive des systèmes initiaux des stations - qui se traduit par une triple rupture d'ancrage : spatio-temporelle (élargissement spatial du territoire de l'offre et des pratiques), fonctionnelle (complexification et mutation des fonctions initiales) et symbolique (changements " d'image ") - se manifeste toutefois avec des intensités et selon des modalités variables en fonction des contextes locaux et de la nature des processus à l'œuvre. II importe donc de disposer d'indicateurs permettant de suivre ces transformations et d'en apprécier l'intensité.

Paramètre classique de l'évaluation des territoires touristiques français, compte tenu de l'ampleur et de l'ancienneté du phénomène ${ }^{2}$, la résidence secondaire offre un intérêt tout particulier pour l'interprétation des trajectoires des stations, dont elle a longtemps représenté l'un des mécanismes fondamentaux de création et de développement, les programmes immobiliers destinés à vendre des logements (la plupart du temps en résidence secondaire) apparaissant indissociables de l'aménagement des domaines skiables dont ils ont permis le financement (François \& Marcelpoil, 2008 ; Arcuset, 2009 ; Vlès 2012 \& 2014). La forte poussée de la résidentialité secondaire en France au

\footnotetext{
${ }^{1}$ Philippe Bachimon, Professeur des universités, Université d'Avignon, UMR Espace-Dev 228 IRD ; Pierre Dérioz, Maître de conférences HDR, Université d'Avignon, UMR Espace-Dev 228 IRD; Vincent Vlès, Professeur des universités, ISTHIA, Université Toulouse-Jean Jaurès, UMR CERTOP 5044. L'expérimentation porte sur quatre cas très différents : une des toutes premières stations de montagne de seconde génération (Gourette, Pyrénées-Atlantiques), deux communes néorésidentielles soumises à l'économie présentielle transfrontalière (Nahuja et Valcebollère, Pyrénées-Orientales), une vallée en restructuration économique de l'ère industrielle à la station de nature, le Haut Vicdessos (Ariège).

2 II y avait en France en 2013, 3,17 millions de résidences secondaires pour 28,08 millions de résidence principales, soit une proportion de $11,3 \%$ de résidences secondaires par rapport aux résidences principales (INSEE, 2014). A la nuance près que $10 \%$ des résidences secondaires ne sont pas possédées par des résidents principaux français, et que les résidents principaux français peuvent avoir des résidences secondaires hors de France (et dans ce cas il sont réputés ne pas avoir de résidence secondaire) ou posséder plusieurs résidences secondaires en France (Bachimon \& alii, 2004)... qui apparaîtront dans les statistiques toujours secondaires... alors qu'elles sont tertiaires, quaternaires. On observe un léger tassement du phénomène depuis le milieu des années 2000 (11,7\% de résidences secondaires en 2004).
} 
début des années 2000, avec des croissances de l'ordre de 100000 unités supplémentaires/an, doit d'ailleurs beaucoup aux acquisitions des étrangers dans les stations - notamment celles des Espagnols dans les stations pyrénéennes -, souvent portées par des démarches d'optimisation fiscale, combinées, parfois, à des projets résidentiels pour la retraite. La part prise aujourd'hui par les résidences secondaires dans la capacité d'accueil des stations pyrénéennes varie certes selon leur nature, mais elle n'est jamais inférieure à $54 \%$, et peut même atteindre $80 \%$, voire plus dans certaines stations de ski (Bachimon, Dérioz, Vlès, 2015). Au-delà de leur rôle historique dans la " fabrication » des stations - entendues comme pôles d'hébergements et d'équipements de loisirs - elles en constituent toujours l'un des composants majeurs pour lequel la dimension collective originelle interfère avec le faisceau des stratégies résidentielles et patrimoniales privées. Les résidences secondaires acquises en station dans le cadre de programmes collectifs en viennent à partager un certain nombre de problématiques avec celles issues de la " secondarisation " des maisons de famille dans les villages de montagne proches, avec lesquels la dilatation spatiale du système des stations a renforcé les interactions.

Souvent étudié sous l'angle des phases spéculatives qui ont conduit à son développement (Barbichon, 1978 ; Dubost, 1998 ; Vlès, 2014), le phénomène des résidences secondaires est à peu près toujours présenté comme divers, mal cerné par les données statistiques disponibles ${ }^{3}$. Diverses études récentes ont aussi insisté sur la faiblesse de leurs taux d'occupation - 42 nuits/an en moyenne selon I'enquête Ipsos Marketing pour Atout France en 2010 (cf., aussi, Atout France, 2013) - et souligné le déficit d'animation des stations induit par cette forte proportion de "lits froids " ou " diffus " échappant à la sphère marchande professionnelle, que les politiques touristiques souhaitent, de manière un peu théorique, réintégrer au sein du marché locatif (Miquel, 2010). Mais peu de travaux se sont intéressés à la complexité croissante qui se cache derrière la définition statistique, qui reflète en réalité la très grande variété des évolutions sociétales contemporaines (familles recomposées, distension entre lieu de travail et lieu de vie, mobilités accrues, ubiquités virtuelles liées notamment au numérique). Il paraît ainsi difficile de continuer à englober sous la seule appellation de " résidences secondaires " les multiples formes que revêt cette modalité résidentielle nouvelle, pour laquelle certains auteurs ont proposé d'autres approches plus compréhensives autour des idées de " multirésidence », de "nomadisme résidentiel» (Bachimon, 2008) ou de "dédoublement résidentiel » (Sansot, 1978).

On démontre ici que le dédoublement résidentiel, saisi dans toute sa complexité et dans toute sa diversité, joue un rôle majeur, à des titres divers, dans le fonctionnement des systèmes touristiques territorialisés qui se sont cristallisés autour des stations de montagne. Ce rôle, par ailleurs, apparaît suffisamment différent selon les contextes locaux (diversité historique et structurelle de la résidence secondaire) et selon les périodes considérées (articulation des cycles du territoire et des cycles de la résidentialité) pour que l'on puisse voir dans le dédoublement résidentiel une clé déterminante pour l'intelligence de la trajectoire de ces systèmes, à la condition de réserver à ce marqueur privilégié une approche particulièrement minutieuse : les travaux de terrain qui nourrissent cette réflexion ont ainsi fait le choix de la grande échelle et reposent sur des enquêtes précises de terrain, des entretiens, des études de cas approfondies (à la parcelle, ou au lot près) réalisées in situ sur plusieurs années (entre 2007 et 2014), dans une perspective comparative qui confronte trois systèmes touristiques différents et hétérogènes du massif pyrénéen : en Cerdagne (Pyrénées-Orientales), en Vicdessos (Ariège) et dans la haute vallée d'Ossau (station de ski de Gourette et station thermale des Eaux-Bonnes, PyrénéesAtlantiques).

\footnotetext{
${ }^{3}$ Les résidences secondaires sont des logements utilisés pour les week-ends, les loisirs ou les vacances pour des saisons touristiques dans les stations touristiques, dans les stations balnéaires, de sports d'hiver, etc. On y classe également les logements meublés, loués ou à louer. Sont inclus dans cette catégorie de logements les cas de multipropriétés ainsi que les gîtes ruraux, les villages de vacances « en dur » et les hôtels résidences de tourisme. Est exclu le logement mobile sédentarisé, caravane ou mobile home installé à demeure sur un terrain. (INSEE 1999). 30\% seulement des 3 millions de résidences secondaires recensées en France remplissent une fonction d'hébergement touristique, saisonnière et occasionnelle, officielle ou non, soit 5 à 6 millions de lits (1,2 millions seulement sont classés ou labellisés) (Direction du tourisme, 2006).
} 
Pourquoi le dédoublement résidentiel est-il un marqueur fondamental des bifurcations de trajectoire des systèmes touristiques?

Depuis les années 1985-1990, le tourisme en montagne en Europe est entré dans une phase de changement global marqué par de multiples facteurs, tels que la concurrence accrue entre les destinations, le vieillissement de la population touristique, l'affaiblissement des dynamiques internes, l'insuffisance des initiatives d'investissement, les déficits chroniques d'exploitation, les exigences croissantes de qualité esthétique et environnementale ou encore la gestion des risques (Perret, 1992 ; Bourdeau, 2007, 2009, 2011, 2013 ; Clarimont \& Vlès, 2008 ; Vlès, 2010, 2012, 2014 ; Bachimon et coll., 2013 ; Botti et coll., 2012 ; Achin et coll., 2013). L'avenir urbain de ces stations, l'adaptation de leur architecture, de leur parc immobilier, leur insertion dans le paysage sont aujourd'hui confrontés à trois mutations dans lesquelles le facteur résidentiel joue un rôle prépondérant.

L'obsolescence du cadre bâti des stations produit un décalage entre l'image de la montagne, la représentation que s'en font les visiteurs et la réalité du paysage, qui déçoit de plus en plus. La manière dont le " touriste " évalue le paysage de la station change et ses perceptions révèlent la conscience qu'il a de cette déconnexion des temps. L'opposition entre le temps figé d'une part - qui type le village ou la station dans la pierre ou le béton, marqué par son urbanisme initial - et le temps en mouvement, celui qui porte l'évolution des pratiques touristiques et de loisirs, prend aujourd'hui un caractère très sensible et touche la grande majorité des lieux de séjours de montagne. Lorsqu'ils sont touristes, les futurs résidents secondaires arrivent sur un site avec des images préétablies et cherchent à adapter leur représentation du village ou de la station à ces images. On sait ainsi que le touriste recherche en station un paysage différent, typé, " authentique ", " traditionnel » (Hatt, $2011: 376$ ) et c'est pourquoi il s'accommode facilement, par exemple, du style néo-savoyard dans les Pyrénées (Bolquère, Eyne, La Pierre Saint-Martin, Saint-Lary-Soulan, etc.), paysage valorisé par les orientations architecturales des stations (Vlès, $2010: 45$ ) ou les brochures touristiques. Le chalet, par exemple, fait partie des images d'Épinal que le touriste s'attend désormais à rencontrer dans toutes les montagnes. Le besoin de dépaysement des citadins, la recherche d'une confrontation permanente à la nouveauté et parfois à " l'authenticité " apparaissent omniprésents dans les enquêtes. Élément paradoxal qui se lit dans le paysage, ces nucléus touristiques de montagne répondent à une logique de plus en plus internationale, normée et standardisée : extension par lotissements, enclaves urbaines porteuses de conformité et normalisées autour d'une réinvention kitsch du bâti, architecture qui n'existe pas par elle-même, affirmation d'une autochtonie factice. Cette urbanisation crée en montagne des enclaves porteuses de conformité, où l'architecture pastiche des nouvelles opérations immobilières est marquée par le conformisme (Vlès, 2014).

Autre infléchissement des trajectoires des aires touristiques en montagne, la " fuite en avant » immobilière constitue une tendance qui a récemment provoqué une bulle immobilière encore non résorbée et qui touche le phénomène de la résidentialité touristique, notamment dans les Pyrénées (Pamir, 2012). D'un côté on assiste à une déprise du tourisme en montagne partout avérée. En 4 ans, la montagne française a perdu près de 6 millions de nuitées, soit $5 \%$ de sa fréquentation. Sur les dix dernières années, ce chiffre est d'environ $10 \%$. L'évolution chaotique liée à des problèmes d'aléas d'enneigement et de températures élevées n'explique pas tout ; les tendances d'évolution de la saison d'été indiquent également une baisse sur l'ensemble des périmètres. Outre les impacts économiques directs, cette érosion de la fréquentation (qui peut atteindre -35 \% entre 2004 et 2007 à Gavarnie, par exemple) implique aussi un désinvestissement dans l'immobilier de loisirs, donc la sortie des hébergements des standards de confort lorsque l'effort de réinvestissement n'a pas été consenti. En conséquence, les attentes des clientèles sont plus difficiles à satisfaire, les biens se louent ou se vendent plus difficilement, ils se disqualifient progressivement : cette spirale place les destinations touristiques de montagne dans une logique "d'exténuation" de l'offre; le phénomène est particulièrement massif dans les Pyrénées (Pamir, 2012). Le processus d'urbanisation liée à la double 
résidentialité avait permis un temps de financer les aménagements collectifs qui agrémentent l'accueil de ces nouveaux habitants, de couvrir les frais et d'amortir les investissements réalisés dans les équipements de loisirs ou le domaine skiable. Dorénavant, cette trajectoire classique des stations est confrontée à la diminution de la demande solvable et à une fréquentation en baisse qui génèrent des contre-performances économiques. Les tentatives de requalification se sont d'ailleurs soldées par deux échecs dans les Pyrénées : celui des opérations de requalification de l'immobilier de loisirs qui n'ont pas pu adapter l'immobilier ancien (particulièrement dans la vallée d'Ossau) et celui des opérations de défiscalisation de l'investissement dans les résidences de tourisme, qui affectent l'immobilier neuf marchand en l'ayant placé, au plus mauvais moment (années 2007-2012), dans une bulle spéculative largement sous influence du marché espagnol (ce phénomène touche l'ensemble des Pyrénées).

Les difficultés de l'immobilier de loisirs sont ainsi devenues la caractéristique principale de l'évolution des systèmes touristiques en montagne pyrénéenne. Or les résidences secondaires y représentent au minimum aujourd'hui entre $54 \%$ et $95 \%$ de leur capacité d'accueil totale.

Par ailleurs, sur la pression des lobbys immobiliers, les gouvernements successifs ont, depuis une douzaine d'années, agi sur une nouvelle forme de résidence secondaire, la résidence de tourisme, faite de logements secondaires mis en location pendant 9 ans, mais dont l'usage peut au-delà revenir totalement à son propriétaire... qui se prive rarement de cette opportunité. Soutenu par défiscalisation, ce dispositif a favorisé dans le massif pyrénéen une bulle immobilière importante : sur les 111000 lits construits en résidence de tourisme en France entre 2000 et 2010 dans les seules zones de montagne, 30000 le sont dans les Pyrénées (Pamir, 2012). C'est beaucoup trop eu égard au potentiel touristique du massif, qui n'est pas de $30 \%$, mais de $10 \%$, et qui reste très marqué par le tourisme de nature, éloigné de l'investissement immobilier. Autre bifurcation de trajectoire touristique des vallées pyrénéennes, la défiscalisation de la résidence secondaire y a produit, par cet effet d'aubaine fiscale, des surcapacités reconnues par les professionnels : en vallée d'Aure, on compte ainsi 10000 lits largement inoccupés et la surcapacité totale actuelle y est évaluée à plus de 30000 lits ; on relève des faillites de résidences, à Gourette, en vallée d'Aure ou en Cerdagne-Haut Conflent et le taux moyen d'achèvement des résidences secondaires en résidence de tourisme, fin 2013, n'était que de $55 \%$ dans les Pyrénées, $45 \%$ dans les Pyrénées-Orientales, contre plus de 98,4\% dans les Alpes.

Ce tableau permet de saisir qu'aujourd'hui l'hybridation en station pyrénéenne s'opère sur l'affirmation de son hyperréalité. Celle-ci se présente comme un amalgame entre le modèle stéréotypique de la " montagne » (elle est alpine, enneigée - c'est-à-dire « blanche " quand elle fait l'objet d'une médiatisation -, saupoudrée de chalets, peuplée de montagnards) et sa copie qui consiste en des lotissements résidentiels aux volets presque toujours clos, en l'enneigement artificiel de pistes devenues ainsi skiables durant l'entière saison... tout un décor kitsch qui en soit devient finalement un environnement vécu par les résidents, qu'ils soient temporaires ou permanents. Cette réalité n'étant d'ailleurs pas nécessairement ressentie comme une falsification, mais plutôt comme un arrangement contingent, un inévitable compromis.

Le phénomène observé est certes contradictoire, car il est à la fois le prérequis urbanistique de la station, la condition sine qua non de son émergence, mais elle est aussi celle de sa sclérose, en terme de déficit d'urbanité, du fait de son très faible taux d'occupation. Elle fonctionne comme un vacuum au cœur du système touristique, en ce sens qu'elle sacralise des espaces en les ôtant au tourisme "banal » (locatif). C'est cela qui fait qu'elle est souvent l'objet de l'intérêt des politiques de réappropriation immobilière, de reconversion fonctionnelle et de rénovation du bâti.

La résidence secondaire peut ainsi être observée comme un indicateur temporel et spatial des trajectoires des stations. Les temporalités de la résidentialité secondaire pyrénéenne se déclinent en phases qui se succèdent, celle du thermalisme (au XIXe siècle), du climatisme (durant toute la première moitié du XXe siècle), celle du ski (durant l'autre moitié) et celle plus actuelle de la pleine nature. 
Sachant qu'il y a des recouvrements de ces cycles et aussi, selon les lieux, des vacances temporelles. Ces temporalités sont inscrites dans le tissu résidentiel secondaire par la localisation, l'urbanistique qui décline un gradient allant de l'individuel à l'immeuble en passant par le lotissement, les modes architecturaux. Tout cela peut se lire aisément dans le paysage à forte résilience comme une mémoire de la station. Avec les oublis des délaissés (parfois un quartier ou toute une station abandonnée) durant les interphases et les rénovations/restaurations dans les phases de patrimonialisation. La Résidence secondaire ancienne pouvant faire l'objet d'une protection... comme mémoire de l'histoire touristique du lieu.

La résidence secondaire est un livre ouvert à la lecture du chercheur. Il y voit le temps long de la trajectoire touristique de la station lorsqu'il la parcourt comme un corpus à ciel ouvert. Mais il en saisit aussi les temporalités plus courtes. Celles des trajectoires de vie de leurs propriétaires, car elles y trouvent une place évolutive (mutatis mutandis) dans leurs trajectoires sociales (familiales en particulier) dans la mesure où elles s'y inscrivent bien souvent dans le moyen terme d'un projet de vie qui va de l'investissement de loisir à la retraite en passant par l'attachement identitaire au pays de ses origines, tandis dans le plus court terme cyclique de l'annualisation des pratiques, celui des rythmes saisonniers, elle entre dans un gradient allant de l'absentéisme chronique à l'occupation maximale en passant par la venue irrégulière et les séjours répétés des passionnés.

Cet emboîtement de temporalités et des spatialités donne à la station sa spécificité. C'est ce caractère protéiforme de la résidence secondaire qui en quelque sorte atténue en large partie l'effet de la standardisation. C'est pour cette raison que la double résidentialité est devenue un objet et un indicateur de ces recherches. La partie suivante donne une illustration de ce type d'approche multiscalaire.

Trois études de cas de bifurcations de territoires touristiques marqués par la " double résidentialité »

La diversité des situations, des usages et des fonctions de la résidence secondaire a des impacts qui débordent les seules mutations paysagères : trois études de cas exhaustives des parcs de résidences montrent que la mutirésidentialité révèle également des normalités d'itinéraires de vie en montagne très variées qui dépassent la seule résidentialité secondaire pour refléter les évolutions sociétales contemporaines disparates et changeantes.

Dans les Pyrénées béarnaises, la vallée d'Ossau est symptomatique des modes de double résidentialité rencontrés dans le reste du massif. La beauté de la vallée, la variété de ses sites, la présence du Parc National et la notoriété des randonnées qui s'y déploient lui ont donné un fort attrait depuis les débuts du Pyrénéisme au XIXe siècle. Aujourd'hui, les sports de neige y constituent une ressource majeure pour le haut Ossau, pratiqués par $88 \%$ des touristes, le ski de fond par $26 \%$ et les autres formes de glisse par $12 \%$ des visiteurs (200 000 nuitées, $5^{\mathrm{e}}$ station du versant français de l'ouest de la chaîne). La très grande majorité de ces visiteurs y ont développé une double résidentialité concentrée à Eaux-Bonnes-Gourette, qui regroupe quatre villages (Assouste, Aas, Eaux-Bonnes et Gourette : 426 habitants en 2012).

La résidence secondaire a fabriqué ces deux stations et son poids y est considérable, même si elle est surtout le fait d'habitations sous la forme d'appartements. À Gourette, seulement 34 des 1320 propriétés $(2,5 \%)$ sont désignées comme des résidences principales par leurs propriétaires. Les résidences secondaires représentent donc plus de $97 \%$ des lots de la station. Neuf lots seulement sont des maisons ou des chalets. Les propriétaires ont en moyenne 63 ans et résident à $73 \%$ en région Aquitaine. Gourette a du mal à renouveler les clientèles qui résident dans ces appartements non marchands (enquête auprès de la direction de la station). Les relevés cadastraux montrent que ce sont en grande majorité des propriétaires qui ont acheté dès l'origine de la station qui transmettent les lots à leurs familles (Loope, 2014). 
Aux Eaux-Bonnes, distante d'une dizaine de kilomètres en aval de Gourette, 5 \% seulement des 534 lots qui la composent sont identifiés comme des résidences principales. Comme pour la station de sports d'hiver, ces propriétaires résident en majorité dans des appartements dont la surface est proche des $60 \mathrm{~m}^{2}$. Leur âge moyen, 68 ans, est à l'image de la station thermale vieillissante. Les résidences secondaires sont en grande majorité des appartements (22 lots sont des maisons ou chalets). Dans les hameaux dispersés du reste du territoire (Aas, Assouste et quelques habitations à l'écart d'un des quatre villages), le taux de résidence principale est plus élevé : $20 \%$. Les résidences secondaires dans cet habitat dispersé représentent 185 lots occupés par des familles nombreuses. En conséquence, les biens ont une surface supérieure à $110 \mathrm{~m}^{2}$. Leurs propriétaires sont presque aussi âgés que dans les deux stations, leur âge moyen est de 65 ans, $78 \%$ viennent de la région Aquitaine, $8 \%$ de la région Poitou-Charentes, 3 \% de la région Rhône-Alpes.

L'hébergement touristique de la commune multipolaire des Eaux-Bonnes-Gourette est donc constitué à $95 \%$ de résidences secondaires. Comme pour l'hébergement marchand, ces lits révèlent des taux d'occupation extrêmement faibles. Pendant l'hiver 2013-2014, leur taux d'occupation était seulement de 8,2 \% et l'été 2013, de 2 \% (Loope, 2014).

Face à des coûts d'investissement et de fonctionnement d'équipements collectifs en constante augmentation - notamment en station de ski, la fréquentation de ce parc immobilier stratégique pose un problème d'avenir essentiel au modèle économique de la vallée : clairement, son modèle de développement est remis fortement en question par l'abandon en cours. Par ailleurs, du fait des sorties du marché locatif des hébergements marchands ${ }^{4}$, les deux stations perdent annuellement $2 \%$ de lits marchands (Comète 2014). Les représentants des copropriétés eux-mêmes n'ont d'ailleurs pas réussi à mettre d'accord l'ensemble des propriétaires afin d'effectuer des rénovations et entretiens jugés jusqu'ici trop coûteux. À Eaux-Bonnes - Gourette, la question de la requalification des lits résidentiels avait déjà été évoquée dans les années 1990. Mais, faute d'intérêt de la part des propriétaires, seuls les espaces publics et les accès ont pu être requalifiés entre 2010 et 2012. Cet investissement sur le domaine public s'est avéré insuffisant pour enrayer le processus de délaissement de la station, dont l'enquête montre combien il est lié au vieillissement de la clientèle des résidents secondaires.

La crise résidentielle - sous fréquentation, perte de lits marchands, absence de requalification du bâti... -, qui pèse sur la capacité de la station à faire face aux coûts d'investissement et de fonctionnement de ses équipements (remontées, neige de culture, équipements d'accompagnement), se combine en effet avec une baisse tendancielle de la fréquentation. Alors même que la Cour des comptes considérait en 2011 que le point d'équilibre financier de la station de Gourette se situait à 360000 jours-skieurs, la fréquentation n'a plus jamais atteint ce niveau depuis 2008-2009 (363 132 js) ${ }^{5}$, la moyenne des six saisons suivantes s'établissant plutôt un peu en dessous des 300000 j-s (avec seulement 267500 j-s pour 2014-2015, en baisse de $16 \%$ par rapport à l'année précédente). En année déficitaire, c'est le Département des Pyrénées-Atlantiques, propriétaire de la station, qui ajuste les comptes de l'établissement public des stations d'altitude (EPSA) par le versement d'une dotation d'équilibre. Des questions liées à la gouvernance de la station et à son articulation avec d'autres instances territoriales viennent donc ici se superposer à cette remise en cause latente du modèle économique de la vallée : la communauté de communes de la vallée d’Ossau, qui rassemble 18 communes (10000 habitants), comporte d'autres communes où les résidences secondaires sont également nombreuses ( $55 \%$ à Louvie-Soubiron, $52 \%$ à Laruns, $47 \%$ à Béost, etc.), ainsi qu'une

\footnotetext{
${ }^{4}$ Pour bénéficier de la défiscalisation, le lit secondaire en résidence de tourisme ne doit être mis en location que pendant 9 ans. Au terme de ce délai, le propriétaire peut à nouveau disposer de son bien comme il l'entend. Par ailleurs, ce phénomène de déperdition s'explique aussi par le vieillissement du parc qui touche la résidence secondaire. La plupart des grands ensembles d'immeubles collectifs construits autour des années 60-70 sont aujourd'hui dégradés du fait de leur âge et du manque d'entretien, leurs appartements deviennent difficile à louer.

${ }^{5}$ Rapport de la Chambre Régionale des Comptes d'Aquitaine sur l'examen des comptes, pour les exercices 2004 à 2007, et de la gestion de 2004 jusqu'à 2010, de l'établissement public des stations d'altitude des Pyrénées-Atlantiques (EPSA), en ligne : https://www.ccomptes.fr/content/.../JF00114614 JF INTERNET1.pdf
} 
deuxième station de sports d'hiver, la petite station d'Artouste (29 200 j-s en 2013-2014, sous gestion déléguée).

Dans les villages de Cerdagne étudiés en 2014 (Valcebollère et Nahuja), le rapport à la station de ski alpin n'est pas absent, même si ces villages n'appartiennent pas formellement à une station. Les deux communes en sont plus ou moins dépendantes du fait de leur topographie, par la route d'accès qui les y relient et la participation ou non à la dette qui grève le domaine alpin du Puigmal auquel elles se rattachent. Ainsi, du fait de son appartenance au syndicat intercommunal gestionnaire de la station du Puigmal, Nahuja, avec seulement 49 habitants permanents, a contracté une dette de 3 millions $€$ à rembourser : c'est la part qui lui revient du déficit accumulé par la station qui, depuis 2013, a été fermée par arrêté préfectoral en raison de son incapacité à honorer ses dettes dans les limites acceptables par la loi. Mais la proximité réelle de cette station a joué comme facteur discriminant positif pour un implantation "espagnole " (qui est essentiellement catalane, puisque composée de ressortissants de la Generalitat) en résidence secondaire. La multirésidentialité a pris deux formes. Celle d'une installation dans les maisons « abandonnées » du vieux village et celle d'un investissement en maisons accolées (adosados).

La résidence secondaire française relève d'un ancrage plus ancien que celle des Barcelonais. La résidence barcelonaise a bénéficié d'un investissement très souvent supérieur. Aussi le bien immobilier barcelonais est-il presque toujours partagé en studios ou multiples appartements qui sont prêtés aux amis, plus rarement loués de manière non déclarative. La rénovation soignée des vieux bâtiments et la décoration kitsch (Vlès, 2014) des nouvelles bâtisses, l'entretien régulier et méticuleux des pelouses par des jardiniers professionnels permettent de reconnaître " au premier coup d'œil » la nationalité du propriétaire, selon un mode d'occupation de l'espace très éloigné de celui du haut Ossau.

L'inversion entre résidentialité réelle et résidentialité légale est rare (un cas identifié, dans chacun des villages étudiés, d'un résident principal déclaré comme résident secondaire), mais, à vrai dire, " le gradient résidentiel » (le présentiel avéré) est particulièrement ouvert et rapproche, aux extrêmes, les situations de certains résidents principaux de celles de résidents secondaires. On peut schématiquement distinguer quatre profils de dédoublement résidentiel.

L'absentéiste serait celui qui ne vient pas une seule fois dans l'année, ou alors de manière tout à fait aléatoire, laissant passer plusieurs mois voire plusieurs années sans revenir, et dont la maison reste inoccupée en quasi permanence. II représente un cas extrême qui tend à faire de son bien un logement vacant, et ce qu'il soit déclaré comme résident principal ou secondaire. En fait, l'absentéisme est le propre des propriétaires entrés dans le très grand âge, lorsque la maladie et les infirmités deviennent un handicap majeur réduisant leur mobilité. Et lorsqu'aussi les enfants, et parfois les petits-enfants, eux-mêmes âgés et retraités, ne viennent plus. L'irrégularité et la rareté extrêmes de la venue apparaissent comme un désinvestissement social avant la mise sur le marché du bien, si des modes $d^{\prime}$ indivision ne viennent pas ralentir ce processus. Cela est ressenti, par les autres résidents, comme une forme de désintérêt : « ils ne viennent jamais », " je ne les ai plus vus depuis des années... " qui s'avère être une source de délégitimation.

Le type du "vacancier régulier " caractérise plutôt le propriétaire barcelonais d'une résidence secondaire. II n'est là qu'à deux courtes périodes, invariables dans l'année, où on le voit « débarquer ». Soit autour " des Rois » (jour de l'an et les 5 jours qui le suivent) pour faire du ski, soit après le 15 août, pour les deux semaines qui précèdent la rentrée des classes, et ce avec l'objectif d'échapper à la chaleur du littoral méditerranéen, littoral où il a quelquefois une villégiature familiale (qui est de fait se "deuxième résidence ", celle de Cerdagne étant sa troisième). À cette catégorie se rattache celle des « Français » qui viennent, en léger décalage, selon une rythmicité comparable, soit aux vacances scolaires de février et entre la mi-juillet et la mi-août. Pour le coup, ces deux groupes ne se croisent quasiment pas et finalement ne se connaissent pas, même s'ils résident durant leur séjour cerdan dans des maisons mitoyennes. 
Le passionné correspond à la catégorie de ceux qui viennent dès qu'il le peuvent pour leurs activités de pleine nature. II vient parfois de Catalogne, mais plus largement du sud de la France. Sur le qui-vive, il " monte " à l'occasion d'une météo propice à l'exercice de son sport préféré, mais aussi pour la cueillette des champignons, pour " être » d'une fête de village... il va parfois aussi s'impliquer plus en avant dans la vie du village en participant aux activités collectives, voire en devenant conseiller municipal.

Le résident à mi-temps représente une catégorie de résidents qui partagent leur temps entre " le haut " et "le bas ». On le retrouve indifféremment dans l'une ou l'autre des catégories résidentielles, avec parfois le cas de couples dont l'un est résident principal du village quand l'autre y est secondaire. Évidemment, le village étant considéré comme lieu de loisir plutôt que de travail (mais il y a des cas de télétravail), c'est en fonction des opportunités climatiques et saisonnières que se fait l'alternance. Les séniors, et plutôt les jeunes retraités constituent le gros de la cohorte. C'est dans leur cas que la catégorisation résidentielle s'avère caduque. Sachant cependant qu'il s'agit de configurations qui peuvent soit perdurer soit être transitoires, car les aléas de la vie et le vieillissement font évoluer, parfois assez brusquement, ce qui en apparence semblait stable. Cette population n'apparaît pas comme " étrangère " aux sédentaires du Haut Plateau. Elle a assez souvent d'ailleurs des liens familiaux avec eux et bénéficie d'une image "positive ». Elle est présente aux moments cruciaux (élections, fêtes du village...), s'investit dans les relations sociales, bénéficie d'un réseau de " connaissances » et amène avec elle un « souffle de l'extérieur ", un exotisme léger, qui est apprécié.

Enfin les résidents à plein temps se recrutent d'abord dans la catégorie des résidents principaux, et en particulier chez les "natifs ", les quasi natifs et les " naturalisés » du fait d'un présentiel qui peut remonter à leurs parents. Mais même si cette catégorie peut se définir comme regroupant : « ceux qui restent quand tous les autres sont partis ", une proportion importante de ses membres n'est pas strictement sédentaire. D'abord ils peuvent être eux-mêmes propriétaires d'une résidence secondaire " en bas ", côté français, mais aussi en Espagne. Ensuite ils quittent les montagnes à la " mauvaise saison ", celle de l'étiage des activités de plein air, soit à l'inter saison. Car le sentiment qui paraît prédominer est qu'il faut s'échapper de temps à autre de ces petits villages " repliés sur eux-mêmes ».

Les multiples déplacements (aller-retour entre deux résidences) qu'induit le dédoublement résidentiel (il y a des cas de triplement déjà évoqués côté espagnol, et plus rares côté français) peuvent s'apparenter à un nomadisme à l'alternance plus ou moins régulière et aux fréquences plus ou moins élevées si l'on se réfère aux cas de figure évoqués précédemment. Si l'on s'en tient à cette métaphore (Bachimon, 2006), deux aspects de la question méritent d'être abordés.

Un jeu de présence absence. L'ubiquité reste sans doute un idéal poursuivi par les acteurs du dédoublement résidentiel. Idéal jamais atteint, si ce n'est par l'artifice de la dématérialisation qui est convoquée pour la surveillance de la villa inoccupée par le biais de l'abonnement à une société de gardiennage et le recours à une caméra vidéo. Mais il l'est aussi par la consultation des webcams des proches stations qui donnent une idée de l'état du domaine skiable ou de l'état de la route des cols. Cette réalité augmentée n'est pas non plus ignorée par le résident une fois qu'il est sur place (le SIG est utilisé communément en randonnée), mais c'est le recours au télétravail qui lui permet plus de souplesse dans ses modes de séjour, et ce surtout depuis que le câblage de la Cerdagne française par la fibre optique, dans les années 2010, à permis d'y améliorer amplement les débits. Le résident temporaire peut ainsi travailler par vidéo, par Email, par texto... et opérer à partir de son sweet home cerdan un peu comme s'il était " en bas " à son bureau; ce qui change la donne en matière de mobilités (elles peuvent être plus fréquentes, plus imprévisibles, moins séquencées...). L'isolat ne perdure plus que dans sa dimension géographique, voire plutôt dans celle de la distance/temps à parcourir pour aller d'une résidence à l'autre, qui devient la principale contrainte ne serait que par les dépenses qu'elle engage. Un subtil jeu de cache-cache spécifie finalement la place qui est donnée à la deuxième résidence. Elle est souvent la réplique idéale à la montagne de la résidence principale en matière d'équipement (et en particulier pour les télécommunications qui les lient l'une à l'autre) et de confort au point de pouvoir donner l'illusion d'un dédoublement (l'ailleurs s'avérant être un autre ici) 
et ce alors que localement elle se particularise plutôt de ses proxémies relationnelles, le pedibus y retrouvant sa fonction sociale en deçà de celle d'un loisir de pleine nature propre à la randonnée.

Le hors saison de la station. "Je ne monte que si la météo est bonne " dit un résident secondaire de la catégorie des passionnés. Remplacer l'astreinte du temps contraint des vacances par celui du temps choisi des loisirs apparaît comme une autoprophétie réalisée, un privilège du résident secondaire. En effet ces hauts lieux, et quoiqu'en disent les institutionnels du tourisme, deviennent, hors saison, inaccessibles à qui ne dispose pas de son propre hébergement. Le randonneur pédestre d'automne ou celui à ski du printemps ne trouve pas à se loger facilement, encore moins à se restaurer et ne peut espérer bénéficier d'équipementiers ou de fournisseurs de loisirs ouverts. Et ce tout particulièrement dans les stations désertées, où la partie équipée de la montagne en dormance prend un air de désolation. Aussi faut-il bénéficier de sa propre autonomie, de celle permise par sa résidence, pour pouvoir accéder à cette montagne réservée plutôt aux connaisseurs. La résidence secondaire est alors le lieu où l'on va stocker son équipement dédié à la pleine nature (VTT, ski, cartes...), où l'on trouvera un couvert pour le soir et un lit pour la nuit, où l'on bénéficiera d'un confort moins spartiate que celui d'un camping-car, où l'on recréera un peu de convivialité en "montant avec ses invités ». C'est sans doute un paradoxe qu'il restera à explorer, mais il se pourrait finalement que la résidence secondaire soit l'un des supports les mieux adaptés, certes sous-employés comme cela a été démontré dans le cas de la haute vallée d'Ossau, des fréquentations de loisir de l'arrière-saison. Une réponse en apparence non marchande (du point de vue de l'hôtellerie, de la restauration ou des loisirs, mais pas du marché immobilier et des autres services... sans parler de la fiscalité) à la question de la désaisonnalisation.

Ainsi la vacuité confère à la résidence secondaire deux qualités reposant sur un paradoxe. Elle offre une disponibilité et confère des facilités d'accès à la montagne à leurs propriétaires qui s'y perçoivent en retour en authentiques permanents, et ce alors même qu'ils y sont peu présents et n'y viennent que par intermittence. Cet écart entre pratique et représentation repose sur une potentialisation, celle d'une liberté dont on pourrait faire usage, qui n'est pas sans susciter localement des malentendus.

La "station " du Vicdessos (Ariège) apparaît tout à fait particulière, puisqu'elle est issue de la politique de reconversion économique vers les activités récréatives et touristiques mises en place à I'initiative de quelques élus de la vallée ${ }^{6}$, dès les années 1990 , pour faire face au traumatisme social majeur que représente la fermeture de l'usine Péchiney d'Auzat (définitive en 2003), après un siècle marqué par la présence industrielle (Dérioz et coll., 2012). La prépondérance contemporaine des résidences secondaires telles que les enregistre l'INSEE au sein de la Communauté de communes d'Auzat et du Vicdessos (figure) correspond donc à un état de fait vieux de près d'un demi-siècle, qu'il faut regarder comme structurel dans ce territoire : avec quelques 1492 résidences secondaires (66\% du parc résidentiel en 2011), le phénomène du dédoublement résidentiel concerne en Vicdessos un potentiel de population dont l'importance numérique, certes difficile à estimer, apparaît supérieure tant à celle des habitants permanents qu'à celle des touristes séjournant (si on évalue leur effectif maximal sur la base du nombre des lits marchands connus.

\footnotetext{
${ }^{6}$ Le principal animateur de cette politique est Bernard Piquemal, maire d'Auzat entre 1985 et 2014, et conseiller général
} depuis 1992. 


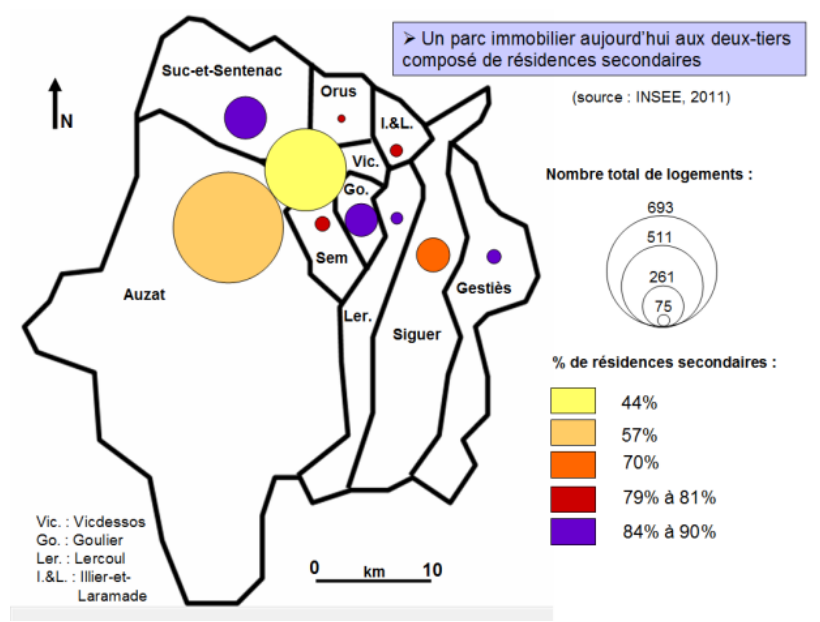

Figure 1 : les résidences secondaires en Vicdessos

Même si ces dernières années semblent indiquer un début d'ouverture sur l'extérieur du marché des résidences secondaires à l'occasion du règlement de certaines successions, leurs propriétaires restent encore aujourd'hui très majoritairement issus de familles enracinées localement : à la maison de famille s'ajoutent parfois des granges restaurées ou des maisons rachetées à des voisins lorsque les enfants d'une même famille ont souhaité s'établir à leur tour dans le village d'origine. II n'est pas rare de recenser dans le même village trois ou quatre maisons relevant d'une même parentèle, auxquelles s'ajoutent aussi celles achetées par des amis venus en vacances et qui ont fini par profiter d'une opportunité pour sauter le pas et s'installer à leur tour (tableau figure 2). À cet enracinement local s'ajoute une fréquente proximité géographique, dans la mesure où les résidences principales de ces personnes se situent majoritairement dans ou à proximité de l'agglomération toulousaine ${ }^{7}$, parfois en Ariège même (Foix, Pamiers, Tarascon-sur-Ariège...). Le grand sud-ouest (Auch, Bordeaux, Montauban...), le Sud-est languedocien et provençal (Marseille) sont également bien représentés, ainsi, sans surprise, que la région parisienne.

Cette proximité géographique, pourtant, n'implique pas nécessairement des durées de présence ou une fréquence des séjours dans les résidences secondaires particulièrement élevées. Le constat dominant est en réalité celui d'une durée de présence sur place assez réduite, centrée sur l'été, avec un spectaculaire pic de fréquentation durant la première quinzaine d'août, qui se trouve logiquement être celle de toutes les festivités locales. À Goulier, où l'on dénombre 39 habitants permanents, le maire considère que la population peut monter à près de 700 personnes durant la première semaine d'août, au moment de la fête du village. Le contraste avec l'ambiance du reste de l'année et particulièrement des mois d'hiver est alors saisissant.

Bien que les volets des résidences secondaires soient souvent fermés, l'enracinement local de leurs propriétaires ne s'en traduit pas moins par un fort désir d'appartenance à ce territoire, revendication identitaire omniprésente non seulement dans leur discours, mais aussi dans la manière dont ils s'impliquent de diverse manière dans la vie locale (figure) : en tant que propriétaires d'un foncier très morcelé dont ils ont hérité en même temps que de leur maison, ils sont nombreux à être membres des quatre Associations Foncières Pastorales mises en place sous l'égide des municipalités (Auzat) et de la communauté de communes pour installer des éleveurs autour des villages et lutter contre leur encerclement par des accrus forestiers plus ou moins denses (Dérioz et coll., 2014). Majoritaires au sein des AFP, à Sem comme à Goulier, Saleix ou Olbier, les résidents secondaires sont aussi parmi les principaux animateurs des associations locales, notamment celles qui prennent en charge l'organisation de la fête du village. Bon nombre d'entre eux, enfin, ont fait le choix de s'inscrire sur les

\footnotetext{
${ }^{7}$ Ces résultats sont très cohérents avec la carte de la location des résidences secondaires des Toulousains établie par l'étude d'Atout France (2010, p.24).
} 
listes électorales - ou même celui de se présenter sur une liste municipale -, et leur vote s'avère souvent déterminant. À Lercoul, par exemple, où ne résident en permanence que 23 personnes, la liste électorale en comptait 92 lors des dernières municipales, qui ont vu l'affrontement de deux listes composées l'une comme l'autre d'un mélange de résidents permanents et secondaires.

\begin{tabular}{|c|c|c|c|c|c|c|}
\hline village & $\begin{array}{c}\text { Nombre de } \\
\text { résidences } \\
\text { secondaire } \\
\mathrm{s}\end{array}$ & $\begin{array}{c}\text { Taux de } \\
\text { résidentialit } \\
\text { é secondaire }\end{array}$ & $\begin{array}{c}\% \text { de } \\
\text { familles } \\
\text { originaires }\end{array}$ & $\begin{array}{c}\% \text { de } \\
\text { familles } \\
\text { ayant connu } \\
\text { le village } \\
\text { par des } \\
\text { amis } \\
\text { originaires }\end{array}$ & $\begin{array}{c}\% \text { de } \\
\text { familles } \\
\text { venues de } \\
\text { l'extérieur } \\
\text { sans lien } \\
\text { local }\end{array}$ & $\begin{array}{c}\% \text { de } \\
\text { familles dont } \\
\text { la résidence } \\
\text { principale se } \\
\text { trouve dans } \\
\text { le } 09 \text { ou le } \\
31\end{array}$ \\
\hline $\begin{array}{c}\text { Olbier } \\
\text { (Auzat) }\end{array}$ & 55 & $90 \%$ & $58 \%$ & $22 \%$ & $20 \%$ & $38 \%$ \\
\hline Sem & 67 & $85 \%$ & $65 \%$ & $7 \%$ & $28 \%$ & $63 \%$ \\
\hline Lercoul & 58 & $86 \%$ & $59 \%$ & $10 \%$ & $\begin{array}{c}26 \% \\
\text { (5\% non } \\
\text { renseignés) }\end{array}$ & $60 \%$ \\
\hline
\end{tabular}

Figure 2 : les résidents secondaires de trois villages du Vicdessos. Source : enquête terrain, 2014.

Les résidents secondaires constituent donc le socle de ce système touristique. C'est avant tout à leur présence que le Vicdessos doit une bonne partie de son animation estivale, au-delà des courtes flambées de fréquentation extérieure liée aux manifestations qui accompagnent le Marathon du Montcalm ${ }^{8}$, ou le passage de l'Ariégeoise ${ }^{9}$. C'est à leur présence que Gourette-Les Eaux-Bonnes a pu croître et prospérer jusqu'à devenir, autrefois et pour quelques années, la première destination des Pyrénées. C'est à leur présence que la Cerdagne et le Capcir ont une économie dont le produit intérieur dépend à $80 \%$ du tourisme et des loisirs. Complexe à évaluer, leur part dans le chiffre d'affaires des diverses activités de pleine nature qui sont proposées n'est peut-être pas prépondérante, mais celle qu'ils prennent dans les rentrées financières des commerces, ou dans la part de vente directe que réalisent les éleveurs locaux ne fait aucun doute. Ces " doubles résidents " participent également directement à l'hébergement touristique, dans la mesure où nombre de résidences secondaires se trouvent souvent prêtées à des amis durant les semaines (plutôt en haute saison estivale ou hivernale) où elles auraient été inoccupées, plus rarement louées. Si réduit que soit le marché immobilier qu'elles représentent, enfin, elles continuent à faire l'objet de transactions, à des prix relativement élevés (proches des prix des centres urbains des métropoles régionales) le phénomène a participé à la rénovation de granges et de bergeries délaissées, assez haut sur les versants (bories, bergeries, granges foraines).

Au-delà des différences relevées entre les sites pyrénéens, un certain nombre de points communs apparaissent : le fort taux de double résidentialité, qui culmine dans les stations de ski ou stations thermales en déshérence, a permis de créer ces "stations » ou de maintenir (artificiellement... c'est une question) en vie des villages, ne serait-ce que par l'entretien du patrimoine; l'économie traditionnelle (agricole, industrielle, ou les deux) a pu être, dans les trois cas et pendant quelques

\footnotetext{
${ }^{8}$ Compétition de trail

${ }^{9}$ Course cycliste, dont les retombées pour le territoire sont évaluées à 300000 euros pour l'édition 2013 (source OT Montagnes de Tarascon et du Vicdessos)
} 
décennies, remplacée par celles de l'immobilier et des services aux loisirs ; les difficultés immobilières sont partout déjà engagées sous des formes variées, mais décelables, liées ici à l'âge des propriétaires, là aux changements des pratiques de loisirs des nouvelles générations. Si à chaque fois le dédoublement résidentiel est apparu comme un "ressort ", voire un "moteur " du système touristique, on voit s'affirmer aujourd'hui quelques-unes de ses fragilités pour l'économie locale. Finalement, les doubles résidents "solides", ceux qui tiennent les villages, ces quelques " passionnés " minoritaires ou "résidents à temps - presque - plein ", ceux-là mêmes qui constituent ce que Laurent Davezies (2004) et Christophe Terrier (2006) nomment "l'économie présentielle » des territoires touristiques pyrénéens sont projectivement les résidents principaux de demain de territoires fragiles.

\section{Bibliographie}

ACHIN C. \& GEORGES-MARCELPOIL E., "Sorties de piste pour la performance touristique des stations de sports d’hiver ", Tourisme \& Territoires, n³, 2013, p. 67-92.

ATOUT FRANCE, 2013, Panorama du tourisme de la montagne, Cahier $n^{\circ} 1$ : l'offre et la fréquentation, col. Observation touristique $n^{\circ}$ 31, Paris, Éditions Atout France, 2013, 11 p.

BACHIMON Ph., "Indicateurs géographiques de la résidence temporaire " in Maby J. (dir.), Objets et indicateurs géographiques, UMR Espace, Actes Avignon, 2003, p. 121-132.

BACHIMON Ph., "Foreign multiresidents in the Luberon region: distinction, assimilation ", Les étrangers dans les campagnes, CERAMAC, $\mathrm{N}^{\circ} 25$, Clermont-Ferrand, 2008, p. 37-52.

BACHIMON Ph., DERIOZ P. \& VLES V., "Quelle place pour les patrimoines culturels et paysagers dans le développement touristique de la Cerdagne ? Pyrénées - France. Rémanences, fragilités contemporaines et interrogations sur la durabilité du modèle ", Ressources patrimoniales et alternatives touristiques: entre oasis et montagne, Edytem, $n^{\circ} 14-2013$, Université de Savoie, 2013, p. 15-24.

BARBICHON G. (dir), BLANCHET A., KARSENTY S. \& PERIANEZ M., L'autre habitat. L'habitat bi-local des résidents secondaires en France, Centre Scientifique et technique du Bâtiment (CSTB), Ministère de l'Environnement et du Cadre de Vie/Direction de la construction, Paris, 1978, 315 p.

BOTTI L, GONCALVES O. \& PEYPOCH N., "Analyse comparative des destinations "neige" pyrénéennes ", Revue de géographie alpine/Journal of alpine research [en ligne], 100-4 | 2012, mis en ligne le 04 janvier 2013,. url : http://rga.revues.org/1843

BOURDEAU Ph, 2007, "L'après-ski a commencé ", dans Philippe Bourdeau, Les sports d'hiver en mutation. Crise ou révolution géoculturelle ?, Cachan, Hermès - Lavoisier, 2007, p. 207-231.

BOURDEAU Ph., « De l'après-ski à l'après-tourisme, une figure de transition pour les alpes ? ", revue de géographie alpine [en ligne], 97-3 | 2009, mis en ligne le 09 décembre 2009, url: http://rga.revues.org/index1049.html

BOURDEAU Ph. \& CHRISTIN Ph., 2011, Le tourisme : émancipation ou contrôle social ?, Paris, Ed. du Croquant, 2011, $288 \mathrm{p}$.

BOURDEAU Ph., "Interroger les mutations et recompositions en cours », Fins (?) et confins du tourisme. Interroger le statut et les pratiques de la recréation contemporaine, Paris, l'Harmattan, 2013, p. 17-39.

CLARIMONT S. \& VLES V., Tourisme durable en montagne : entre discours et pratiques. Préambule 
et introduction, Paris, Editions Afnor, 2008, 226 p.

DAVEZIES L., « Temps de la production et temps de la consommation : les nouveaux aménageurs du territoire ? ", Futuribles, $n^{\circ} 295,2004$, p. 43-56.

DERIOZ P., BACHIMON P. \& LOIREAU M., 2015, " Mise en scène du paysage montagnard et valorisation sélective des patrimoines dans une vallée pyrénéenne en reconversion économique (Vicdessos, Ariège) ", Projets de paysage, $n^{\circ}$ 11, 2015, url : http://www.projetsdepaysage.fr/fr/mise_en_scene_du_paysage_montagnard_et_valorisation_se lective_des_patrimoines_dans_une_vallee_pyreneenne_en_reconversion_economique_]

DERIOZ P., LOIREAU M., BACHIMON Ph. \& CANCEL E., "Quelle place pour les activités pastorales dans la reconversion économique du Vicdessos (Pyrénées ariégeoises) ? ", in TURQUIN O. \& MOUNET C. (Dir.) "Espaces et acteurs pastoraux : entre pastoralisme et pastoralité ", Revue de Géographie Alpine / Journal of alpine research, $n^{\circ}$ 2014-2, url: http://rga.revues.org/2287]

DERIOZ P., BACHIMON Ph., LOIREAU M., LAQUES A.-E. \& DESSAY N., " La mise en tourisme d'un territoire montagnard fragilisé. Sports de nature et patrimoine au cœur du projet de développement et de la politique de communication en Vicdessos (Ariège, France) ", Revue des Régions Arides, $n^{\circ} 28 \quad\left(2 / 2012, \quad n^{\circ}\right.$ spécial), 2012, p. 17-31. url: http://www.ira.agrinet.tn/imgcommon/files/Colloque LOTH 2012.pdf]

DERIOZ P., L'apparence des choses. Analyser les paysages pour comprendre les systèmes territoriaux, Habilitation à Diriger des Recherches, E.N.S. de Lyon, 2012, 348 p.

DI MEO G., Géographie sociale et territoires, coll. Géographie, Nathan Université, 1998, 317 p.

DUBOST Fr. (dir.), L'autre maison. La « résidence secondaire ", refuge des générations. Autrement, collection mutations, $n^{\circ} 178$, avril 1998, $183 \mathrm{p}$.

DUBOST Fr., BONNAIN R., CICE Ch., CLOAREC J., PERROT M. \& al. Les résidences secondaires. Nouvelles orientations. Rapport final à la DATAR, Groupe de prospective sur l'avenir, 1995, 348 p.

FABLET G., Les dynamiques foncières et immobilières comme clefs de lecture du devenir des stations touristiques. Contexte, enjeux et perspectives de recherche, Grenoble, Irstea, 2011, 45 p.

FRANCOIS H., De la station ressource pour le territoire au territoire ressource pour la station. Le cas des stations de moyenne montagne périurbaines de Grenoble, Thèse de Doctorat, Université Joseph Fourier, Grenoble, 2007, 352 p.

FROCHOT I. \& KREZIAK D., " Customers perceptions of ski resorts' images: implications for resorts' positioning strategies ", Tourism and HospitalityResearch, vol. 8(4), 2008, p. 298-308.

HATT E., VLES V., CLARIMONT S. \& DELETRAZ G., " Retour sur images. Les stations touristiques de Seignosse et Gourette sous le regard des touristes ", espacestemps.net, textuel, 10.10.2011, http://www. espacestemps.net/document9036.html

HATT E., Requalifier les stations touristiques contemporaines : une approche des espaces publics. Application à Gourette et Seignosse-océan, thèse de doctorat d'aménagement et urbanisme, Pau, Université de Pau et des Pays de l'Adour, 2011, 644 p.

KLEIN J.-L., TARDIF C. CARRIERE J. \& LEVESQUE B., « Les milieux d'appartenance au Québec. Une perspective méthodologique ", in F. Lasserre \& A. Lechaume (dir.), Le territoire pensé. Géographie des représentations territoriales, introduction, coll. Géographie contemporaine, Presses de I'Université du Québec, Sainte-Foy, 2003, p. 133-163.

LACOUR Cl., " La tectonique des territoires : d'une métaphore à une théorisation ", in PECQUEUR B. (éd.), Dynamiques territoriales et mutations économiques, chapitre 1, coll. Géographies en liberté, éd. L'Harmattan, Paris, 1996, p. 25-48.

LARDON S. \& PIVETEAU V., « Méthodologie de diagnostic pour le projet de territoire : une approche 
par les modèles spatiaux ", Revue de Géographie de Lyon, vol. 80, n², Le diagnostic des territoires, 2005, p. 75-90.

LIMA S., " La " fabrique » de territoires politiques au Mali. Quelles articulations entre les espaces de vie des habitants et les mailles fonctionnelles de l'Etat ", in A. BLETON-RUGET A., COMMERCON N. \& GONOD P., Territoires institutionnels, territoires fonctionnels, Institut de Recherche du Val de Saône-Mâconnais, Mâcon, 2006, p. 187-197.

LOOPE J., Diagnostic et stratégie de développement de l'hébergement touristique à Eaux-BonnesGourette, Mémoire de master 2 "Loisirs, tourisme et développement territorial » sous la direction de Vincent Vlès, Université de Pau et des Pays de l'Adour, 2014, 75 p.

LEVEQUE Ch., MUXART T., ABBADIE L., WEIL A. \& VAN DER LEEUW S., "L'anthroposystème : entité structurelle et fonctionnelle des interactions sociétés - milieux ", in Ch. LEVEQUE CH. \& VAN DER LEEUW S. (dir.), quelles natures voulons-nous ?, Elsevier, Paris, 2003, p. 110-129.

MOINE A., Le territoire : comment observer un système complexe, coll. Itinéraires géographiques, éd. L'Harmattan, Paris, 2007, 176 p.

MIQUEL Fr., MOUGE J. \& RIBIERE G., La réhabilitation de l'immobilier de loisirs en France, Paris, ministère de l'Écologie, de l'Énergie, du Développement durable et de la mer, 2010, 53 p.

PAMIR Conseil, Bilan et perspectives des résidences de tourisme. Massif des Pyrénées. Synthèse 2012, Direction Départementale des Territoires des Hautes-Pyrénées, $1^{\mathrm{er}}$ mars 2012, 34 p.

PERRET J., Le développement local - les stations de sports d'hiver, Grenoble, Université PMFCemagref, thèse de doctorat en économie du développement, 1992, 302 p.

ROLLAND-MAY Ch., Évaluation des territoires. Concepts, modèles, méthodes, Hermès Science, Paris, 2000, $381 \mathrm{p}$.

STROMBONI M., Valorisation de la ressource paysagère et place de la filière touristique dans le système territorial du Haut-Vicdessos, mémoire Master 1 " Géomatique et conduite de projets territoriaux ", Université d'Avignon et des Pays de Vaucluse, 2012, 106 p.

TERRIER Ch., "L'économie présentielle, un outil de gestion du territoire », Cahier Espaces n 90, Paris, éditions espace tourisme \& loisirs, 2006.

VANIER M., "La petite fabrique de territoires, en Rhône-Alpes : acteurs, mythes et pratiques ", Revue de Géographie de Lyon, vol. 70, n²-95, Les nouvelles mailles du pouvoir local, 1995, p. 93103.

VANIER M. \& SCHERRER F., "Les nouvelles mailles du pouvoir local », Géocarrefour, vol.70, n², 1995, p. 91-92.

VLES V., "Du moderne au pastiche : questionnement sur l'urbanisme des stations de ski et d'alpinisme ", Mondes du tourisme, $\mathrm{n}^{\circ} 1$, juin 2010, p. 39-48 : http://www.revueespaces.com/librairie/7743//urbanisme-architecture-stations-sports-hiver-stationsmontagne.html

VLES V., "Ski resorts in crisis and territorial construction in french Catalonia ", Revue de géographie alpine/Journal of alpine research : http://rga.revues.org/1824; 28 décembre 2012 doi : 10.4000/rga.1824

VLES V., Métastations. Mutations urbaines des stations de montagne. Un regard pyrénéen, Presses universitaires de Bordeaux, 2014, 191 p.

VLES V., "Intermunicipal cooperation and tourism : New local roots», in Tourism, recreation and Regional Development : Perspectives from France and Abroad, DISSART J-C., DEHEZ J., MARSAT JB. (Eds). London : Ashgate, 2015, p. 1-22. 
